\title{
Risk factors for symptomatic and asymptomatic norovirus infection in the community
}

\author{
G. PHILLIPS*, C. C. TAM, L. C. RODRIGUES AND B. LOPMAN \\ Department of Epidemiology and Population Health, London School of Hygiene and Tropical Medicine, \\ London, $U K$
}

(Accepted 10 November 2010; first published online 17 December 2010)

\section{SUMMARY}

The objective of this study was to investigate risk factors for norovirus-associated infectious intestinal disease (IID) and asymptomatic norovirus infection. Individuals with IID and healthy controls were recruited in a community-based study in England (1993-1996). This is the first risk-factor study to use viral load measurements, generated by real-time RT-PCR, to identify cases of norovirus-associated IID and asymptomatic infections. Using multivariable logistic regression the main risk factor identified for norovirus-associated IID was contact with a person with IID symptoms. Infectious contacts accounted for $54 \%$ of norovirus cases in young children and $39 \%$ of norovirus cases in older children and adults. For young children, contacts outside the household presented the highest risk; for older children and adults, the highest risk was associated with child contacts inside the household. Foreign travel and consumption of shellfish increased the risk of norovirus-associated IID. Lifestyle and dietary factors were associated with a decreased risk of both norovirus-associated IID and asymptomatic infection. No risk factors were identified for asymptomatic norovirus infection.

Key words: Gastrointestinal infections, infectious disease epidemiology, Norwalk agent and related viruses.

\section{INTRODUCTION}

Norovirus is the most common cause of infectious intestinal disease (IID) in the community in highincome countries [1-4]. Norovirus infection has also been identified in a substantial proportion of individuals with no IID symptoms in several community-based studies, with prevelances of up to $16 \%$ reported in high-income countries [1, 5-7]. Volunteer studies have demonstrated the occurrence of

* Author for correspondence: Dr G. Phillips, Institute for Health and Human Development, University of East London, Water Lane, London E15 4LZ, UK.

(Email: g.phillips@uel.ac.uk) norovirus infection with no concurrent IID symptoms after experimental inoculation $[8,9]$.

While there is a large body of epidemiological evidence on the modes of transmission and risk factors for norovirus-associated IID in outbreak settings, relatively few studies have examined risk factors in the community across all age groups $[6,10,11]$, and risk factors for norovirus infection without IID symptoms (hereafter referred to as 'asymptomatic norovirus infection') have not been investigated. The aim of this study was to identify risk factors for both symptomatic and asymptomatic norovirus infection in the community.

The data are taken from the IID study in England; a previous analysis of data from this study population 
examined risk factors for norovirus-associated IID, diagnosed by electron microscopy, in individuals aged $\geqslant 5$ years [12]. The specimens from the IID study were archived and have been retested using more sensitive molecular methods to detect norovirus [13-15]. We used the results from this retesting to identify cases of norovirus-associated IID and asymptomatic norovirus infections, and looked for risk factors for both symptomatic and asymptomatic norovirus infection, across all age groups.

\section{METHODS}

\section{Recruitment}

IID cases and healthy controls were recruited in the IID study in England, conducted between 1993 and 1996 [12]. IID cases had acute diarrhoea or vomiting and were recruited from either: (i) a prospectively followed cohort in the community; or (ii) after consultation with their general practitioner (GP) for IID. Healthy controls, with no recent history of IID, were recruited from within the community cohort or from the registration lists of participating general practices [16]. Controls were recruited concurrently to IID cases and matched by age, sex and GP practice. No participants were recruited in relation to recognized IID outbreaks, they were only sporadic cases occurring in the community cohort or who presented to a GP. IID cases and controls provided written, informed consent at the time of recruitment.

\section{Epidemiological data}

IID cases and controls completed a risk-factor questionnaire, providing information on social and demographic characteristics; household characteristics and daily activities; and specific exposures related to IID. Adults completed the questionnaire themselves; a parent or guardian completed the questionnaire on behalf of children aged $<16$ years [16]. The specific questionnaire items used in this analysis are given in Supplementary File 1 (available online). The variables created from these questionnaire items are shown in Table 1.

Hand hygiene was captured as the response of the person in the household responsible for food shopping and preparation to the statement: 'It doesn't matter whether you wash your hands or not before handling food' (response options were agree/disagree/ don't know). Foreign travel was defined as spending one or more nights outside the UK in the past 10 days.
Table 1. Conceptual framework for analysis of risk factors for norovirus-associated IID and asymptomatic norovirus infection

\begin{tabular}{ll}
\hline \hline Level & Variable \\
\hline Distal factors & Age \\
& Sex \\
& Social class* \\
& Household size (number of people) \\
& Household age structure (number of \\
& children aged <5 years) \\
& Household crowding (number of people \\
& per room) \\
& Baby in nappies living in the household $\dagger$ \\
& Pet ownership \\
& Sharing a bathroom or toilet with another \\
& household \\
& Nursery/day-care attendance $\dagger$ \\
& Breast feeding§ \\
& Hand hygiene $\|$ \\
Intermediate & Water sports \\
factors & Foreign travel \\
& Animal contact \\
Proximal & Food (raw fruit/vegetables/shellfish/meals \\
factors & prepared outside home) \\
& Household infectious contact \\
& Infectious contact outside the household \\
\hline \hline &
\end{tabular}

* Social class was based on occupation of the wage-earner in the household [51].

$\dagger$ Investigated as a risk factor only for children aged $\geqslant 5$ years and adults.

$\$$ Investigated as a risk factor only for children aged $<5$ years.

$\S$ Investigated as a risk factor only for infants aged $<1$ year.

|| Measured as the response of the person in the household responsible for food shopping and preparation to the statement 'It doesn't matter whether you wash your hands or not before handling food' - response options were agree/ disagree/don't know.

Potentially infectious contacts were defined as anyone with IID symptoms; these infectious contacts were not enrolled in the study and therefore it was not possible to determine the pathogen causing their symptoms.

\section{Specimens and testing}

IID cases provided a faecal specimen during acute illness and controls provided a specimen at recruitment. Norovirus was detected by electron microscopy in the original study and all specimens with sufficient volume remaining after testing were archived in frozen storage [15]. All specimens, including those 
previously positive by electron microscopy, were subsequently retested for norovirus using a more sensitive semi-quantitative real-time reverse transcriptionpolymerase chain reaction (RT-PCR) [13].

There are five norovirus genogroups; noroviruses from two of these genogroups (I and II) cause illness in humans. For this analysis, IID cases who were infected with genogroup II noroviruses were classified as a case of norovirus-associated IID if they had a faecal norovirus viral load, measured by semiquantitative real-time RT-PCR, above age-specific viral load cut-offs as defined in a previous study [14]. No cut-off has been defined for genogroup I noroviruses [14], so only those IID cases with genogroup I norovirus infections detected by electron microscopy and confirmed as positive by norovirus realtime RT-PCR were included as norovirus cases, because the detection limit of electron microscopy is at high viral loads that are associated with norovirus disease $[8,17,18]$. These criteria were used to identify IID cases with norovirus-associated IID, to ensure that those IID cases shedding norovirus at low concentration, with disease caused by another pathogen, were not included in the analysis.

\section{Inclusion criteria and case definition}

IID cases with disease attributed to norovirus according to the above criteria were classified as 'norovirus cases'.

Controls who had been free of diarrhoea and vomiting for at least 10 days were eligible for inclusion in this study; they were considered asymptomatic with respect to IID, although they may have experienced other symptoms during the 10-day exclusion period. Controls testing positive for norovirus by either electron microscopy or semi-quantitative real-time RT-PCR, or both, were classified as 'asymptomatic norovirus infections'. Controls testing negative for norovirus by both electron microscopy and semi-quantitative real-time RT-PCR were classified as 'norovirus negative controls'.

Recruitment, testing and classification of norovirus status are summarized in Supplementary File 2.

\section{Conceptual framework}

We investigated risk factors for norovirus-associated IID, by comparing norovirus cases to norovirus negative controls, and investigated risk factors for asymptomatic norovirus infection, by comparing asymptomatic norovirus infections to norovirus negative controls.

We investigated exposures which are recognized to be associated with norovirus-associated IID, or which may be involved in these transmission routes. A hierarchical conceptual framework [19] was used to investigate risk factors, separately, for norovirusassociated IID and asymptomatic norovirus infection (Table 1; Supplementary File 3 provides published references to support the conceptual framework). The conceptual framework had three levels: (i) distal factors, which are general characteristics and long-term behaviours, e.g. socioeconomic and demographic information; (ii) intermediate factors, which are specific behaviours that may increase the risk of exposure for a short time but are not necessarily always a direct source of infection; and (iii) proximal factors which are a direct source of infection. Reporting of intermediate and proximal risk factors was limited to the previous 10 days before symptom onset for norovirus cases, and the 10 days before questionnaire completion for asymptomatic norovirus infections and norovirus negative controls.

The intermediate and proximal risk factor models were adjusted for higher level variables in the conceptual framework (i.e. distal factors for the intermediate risk-factor model and both distal and intermediate factors for the proximal risk-factor model). Indicator variables for GP practice and month since the beginning of the study were added to the proximal risk-factor models that included infectious contacts, to account for both geographical and temporal variation in norovirus transmission. Children aged $<5$ years and older children (aged 5-15 years) and adults were analysed separately.

There were insufficient numbers of norovirus cases with matched controls from the original recruitment, who were norovirus negative by real-time RT-PCR, to allow a matched analysis of risk factors for norovirus-associated IID. We used an unmatched analysis, but, in addition to including indicators for time of recruitment (month since beginning of the study) and GP practice, we adjusted for the other matching factors, sex and age, to account for similarities between norovirus cases and norovirus negative controls introduced during recruitment [20].

\section{Dealing with missing values}

Two separate analyses were performed using: (i) all participants, creating a categorical indicator for 
missing responses (missing indicator); and (ii) all participants with missing responses imputed (multiple imputation).

Missing responses in the explanatory variables included in the conceptual framework were imputed, using imputation by chained equations, in Stata v. $10.1[21,22]$. The imputation prediction model, which was used to select the most likely value for a missing response, included all variables in the conceptual framework. In addition, indicator variables for the following characteristics were included in the multiple imputation prediction model: GP practice; month since the beginning of the study; the route of recruitment into the study (community cohort or GP registration list); norovirus infection status; and the norovirus season in England and Wales during 1993-1996 (defined in Supplementary File 4). There was no missing data in these indicator variables; they only informed the imputation of missing responses in the explanatory variables from the conceptual framework. Twenty imputed datasets were created and analysed together.

\section{Regression modelling}

The entire model selection process was performed separately on the missing indicator and multiple imputation datasets.

Standard logistic regression models were fitted using Stata v. 10.1 [22]. The imputed datasets were analysed using the 'ICE' suite of commands [21], in which the logistic regression model is fitted separately to each of the 20 imputed datasets. The results are then combined, to give one-point estimate for each odds ratio, and standard errors that take account of uncertainty in both the multiple imputation process and the standard regression [21].

For each analysis, the distal risk-factor model was fitted first and any variables with a $P$ value $<0 \cdot 1$ were selected for inclusion in the final model, for further investigation of their effects. This variable selection process was repeated for the intermediate and proximal risk-factor models. The results presented include variables with a $P$ value $<0 \cdot 1$ in the final model.

\section{Population attributable fractions (PAFs)}

PAFs were calculated in Stata v. 10.1 from the final multiple imputation regression models, using the AFLOGIT programme [23] within the ICE programme, with user-defined code (I. White, personal communication).

\section{RESULTS}

There were 237 norovirus cases, 344 asymptomatic norovirus infections and 1721 norovirus negative controls available for the analysis. Results from the mulitple imputation models are presented in Tables 2-4. The final model for the missing indicator analysis of risk factors for norovirus-associated IID was identical to that from the multiple imputation analysis, with very similar effect estimates. Results from the missing indicator analysis of risk factors for norovirus-associated IID are provided in Supplementary File 5.

\section{Risk factors for norovirus-associated IID in children aged $<5$ years}

Children from households where the main wageearner had a manual or unskilled occupation had more than twice the odds of norovirus-associated IID compared to those from non-manual occupation social classes (Table 2). Recent foreign travel greatly increased the odds of norovirus-associated IID (Table 2). Norovirus-associated IID was strongly associated with contact with individuals with IID symptoms (Table 2). While the odds were much higher for contacts outside the household (Table 2), they accounted for a similar proportion of norovirusassociated IID episodes as infectious contacts inside the household [household infectious contacts: PAF $33 \%$ [95\% confidence interval (CI) 19-48]; infectious contacts outside the household: PAF 32\% (95\% CI 20-44)]. Taken together, infectious contacts inside or outside the household accounted for $54 \%$ of norovirus-associated IID episodes in children aged $<5$ years (95\% CI 42-66). For infectious contacts inside the household, the odds of norovirus-associated IID were higher when the infectious contact was another young child, compared to infectious contacts aged $\geqslant 5$ years, and the odds increased slightly with the number of infectious household contacts (Table 4).

Eating fruit and raw vegetables was associated with lower odds of norovirus-associated IID in children aged $<5$ years, as was contact with animals during this time.

\section{Risk factors for norovirus-associated IID in older children and adults}

Older children (aged 5-15 years) and adults living in a household with a baby in nappies were at three times the odds of norovirus-associated IID (Table 3). The 
Table 2. Risk factors for norovirus-associated IID in children aged <5 years in England, 1993-1996

\begin{tabular}{|c|c|c|c|c|c|}
\hline & \multicolumn{2}{|c|}{ Exposure prevalence $(\%)$} & \multirow[b]{2}{*}{$\begin{array}{l}\text { Odds } \\
\text { ratio* }\end{array}$} & \multirow[b]{2}{*}{$95 \% \mathrm{CI}$} & \multirow[b]{2}{*}{$P$ value $\dagger$} \\
\hline & $\begin{array}{l}\text { Norovirus } \\
\text { cases }\end{array}$ & $\begin{array}{l}\text { Norovirus } \\
\text { negative } \\
\text { controls }\end{array}$ & & & \\
\hline Total & 81 & 461 & & & \\
\hline \multicolumn{6}{|l|}{ Social class } \\
\hline Non-manual & $35 \cdot 8$ & $56 \cdot 8$ & $1 \cdot 0$ & & \\
\hline Manual/unskilled & $50 \cdot 6$ & $35 \cdot 6$ & $2 \cdot 3$ & $1 \cdot 4-3 \cdot 9$ & $0 \cdot 002$ \\
\hline Military & $1 \cdot 2$ & $0 \cdot 9$ & $2 \cdot 3$ & $0 \cdot 2-22 \cdot 1$ & $0 \cdot 46$ \\
\hline Housewife/student/carer & $6 \cdot 2$ & $2 \cdot 6$ & $4 \cdot 1$ & $1 \cdot 4-12 \cdot 3$ & $0 \cdot 01$ \\
\hline Missing & $6 \cdot 2$ & $4 \cdot 1$ & & & \\
\hline Foreign travel & $2 \cdot 5$ & $0 \cdot 9$ & $6 \cdot 6$ & $0 \cdot 9-47 \cdot 3$ & $0 \cdot 06$ \\
\hline Missing & $1 \cdot 2$ & $1 \cdot 3$ & & & \\
\hline Animal contact & $32 \cdot 1$ & $44 \cdot 5$ & $0 \cdot 6$ & $0 \cdot 3-1 \cdot 0$ & $0 \cdot 06$ \\
\hline Not sure & $1 \cdot 2$ & $2 \cdot 0$ & $0 \cdot 5$ & $0 \cdot 1-4 \cdot 8$ & $0 \cdot 57$ \\
\hline Missing & $1 \cdot 2$ & $3 \cdot 0$ & & & \\
\hline Raw fruit eaten & $60 \cdot 5$ & $75 \cdot 9$ & $0 \cdot 5$ & $0 \cdot 3-0 \cdot 8$ & $0 \cdot 007$ \\
\hline $\begin{array}{l}\text { Pre-prepared raw salad or } \\
\text { vegetables eaten }\end{array}$ & $1 \cdot 2$ & $7 \cdot 2$ & $0 \cdot 2$ & $0 \cdot 0-1 \cdot 3$ & $0 \cdot 08$ \\
\hline Household infectious contact & $39 \cdot 5$ & $9 \cdot 3$ & $5 \cdot 7$ & $2 \cdot 0-16 \cdot 2$ & $0 \cdot 001$ \\
\hline Not sure & $2 \cdot 5$ & $1 \cdot 3$ & $3 \cdot 6$ & $0 \cdot 3-47 \cdot 9$ & $0 \cdot 33$ \\
\hline Missing & $9 \cdot 9$ & $3 \cdot 3$ & & & \\
\hline Infectious contact outside the household & $34 \cdot 6$ & $6 \cdot 7$ & $33 \cdot 9$ & $9 \cdot 5-121 \cdot 1$ & $<0 \cdot 001$ \\
\hline Not sure & $16 \cdot 0$ & $13 \cdot 9$ & $4 \cdot 4$ & $1 \cdot 5-13 \cdot 3$ & $0 \cdot 009$ \\
\hline Missing & $1 \cdot 2$ & $0 \cdot 7$ & & & \\
\hline
\end{tabular}

CI, Confidence interval.

* All odds ratios are from multiple imputation models and are adjusted for age and sex; odds ratios for intermediate and proximal risk factors are adjusted for higher level variables in the conceptual framework that were included in the final model. $\dagger P$ values are from a Wald test of regression coefficients.

odds were also increased for older children and adults living in households where at least one member was a child aged $<5$ years (Table 3 ) and these two exposures accounted for similar proportions of norovirusassociated IID episodes [living in a household with children aged $<5$ years: PAF $20 \%(95 \%$ CI $10-30)$; living with a baby: PAF $16 \%(95 \%$ CI $8-25)]$.

Individuals reporting recent contact with a person with IID symptoms were at increased odds of norovirus-associated IID, but the risk was similar for household contacts and contacts outside the household (Table 3). Infectious contacts accounted for almost half of norovirus-associated IID in older children and adults [household infectious contacts: PAF $24 \%$ (95\% CI 13-33); infectious contacts outside the household: PAF $22 \%$ (95\% CI 13-30); combined PAF $39 \%$ (95\% CI 29-49)]. For infectious contacts inside the household, the odds of norovirus-associated IID were much higher if the infectious contact was a child aged $<5$ years and the odds increased with the number of infectious contacts (Table 4).

Foreign travel and eating shellfish increased the odds of norovirus-associated IID in older children and adults (Table 3). Shellfish consumption accounted for a small proportion of norovirus-associated IID episodes in older children and adults (oysters and whelks or winkles: PAF $2 \%, 95 \%$ CI $0-4$ ).

Consumption of fruit, recent participation in water sports and contact with animals were associated with lower odds of norovirus-associated IID (Table 3).

\section{Risk factors for asymptomatic norovirus infection}

Females had slightly increased odds of asymptomatic norovirus infection [children aged $<5$ years, odds ratio (OR) $1 \cdot 4,95 \%$ CI $1 \cdot 0-1 \cdot 9, P=0.07]$; older children and adults (OR 1.4, 95\% CI 1.0-2.0; 
Table 3. Risk factors for norovirus-associated IID in older children (aged 5-15 years) and adults in England, 1993-1996

\begin{tabular}{|c|c|c|c|c|c|}
\hline & \multicolumn{2}{|c|}{ Exposure prevalence $(\%)$} & \multirow[b]{2}{*}{$\begin{array}{l}\text { Odds } \\
\text { ratio* }\end{array}$} & \multirow[b]{2}{*}{$95 \% \mathrm{CI}$} & \multirow[b]{2}{*}{$P$ value } \\
\hline & $\begin{array}{l}\text { Norovirus } \\
\text { cases }\end{array}$ & $\begin{array}{l}\text { Norovirus } \\
\text { negative } \\
\text { controls }\end{array}$ & & & \\
\hline Total & 156 & 1260 & & & \\
\hline \multicolumn{6}{|l|}{ Household structure } \\
\hline Single person household & $4 \cdot 5$ & $6 \cdot 9$ & $1 \cdot 1$ & $0 \cdot 5-2 \cdot 3$ & $0 \cdot 86$ \\
\hline Adults and children aged $\geqslant 5$ years only & $53 \cdot 8$ & $71 \cdot 0$ & $1 \cdot 0$ & & \\
\hline 1 or more child(ren) aged $<5$ years & $30 \cdot 1$ & $14 \cdot 1$ & $2 \cdot 5$ & $1 \cdot 6-4 \cdot 0$ & $<0 \cdot 001$ \\
\hline Missing & $11 \cdot 5$ & $8 \cdot 0$ & & & \\
\hline Baby wearing nappies in the household & $25 \cdot 0$ & $9 \cdot 0$ & $2 \cdot 9$ & $1 \cdot 8-4 \cdot 6$ & $<0 \cdot 001$ \\
\hline Missing & $1 \cdot 9$ & $2 \cdot 9$ & & & \\
\hline Water sports in last 10 days & $10 \cdot 3$ & $17 \cdot 9$ & $0 \cdot 4$ & $0 \cdot 2-0 \cdot 8$ & $0 \cdot 009$ \\
\hline Missing & $7 \cdot 7$ & $6 \cdot 1$ & & & \\
\hline Foreign travel & $7 \cdot 1$ & $2 \cdot 5$ & $3 \cdot 3$ & $1 \cdot 5-7 \cdot 3$ & $0 \cdot 004$ \\
\hline Missing & $1 \cdot 9$ & $2 \cdot 4$ & & & \\
\hline Animal contact & $19 \cdot 2$ & $36 \cdot 3$ & $0 \cdot 4$ & $0 \cdot 3-0 \cdot 7$ & $<0 \cdot 001$ \\
\hline Not sure & $3 \cdot 8$ & $1 \cdot 0$ & $3 \cdot 3$ & $1 \cdot 0-10 \cdot 7$ & $0 \cdot 05$ \\
\hline Missing & $5 \cdot 8$ & $3 \cdot 2$ & & & \\
\hline Oysters eaten & 1.9 & $0 \cdot 1$ & $18 \cdot 3$ & $1 \cdot 5-226 \cdot 6$ & 0.02 \\
\hline Whelks/winkles eaten & 1.9 & $0 \cdot 1$ & $20 \cdot 5$ & $1 \cdot 6-265 \cdot 7$ & $0 \cdot 02$ \\
\hline Raw fruit eaten & $71 \cdot 8$ & $82 \cdot 5$ & $0 \cdot 6$ & $0 \cdot 4-0 \cdot 8$ & $0 \cdot 006$ \\
\hline Household infectious contact & $26 \cdot 9$ & $6 \cdot 7$ & $4 \cdot 9$ & $2 \cdot 7-8 \cdot 8$ & $<0 \cdot 001$ \\
\hline Not sure & $4 \cdot 5$ & $2 \cdot 5$ & $2 \cdot 2$ & $0 \cdot 7-6 \cdot 9$ & $0 \cdot 16$ \\
\hline Missing & $12 \cdot 8$ & $9 \cdot 8$ & & & \\
\hline Infectious contact outside the household & $26 \cdot 9$ & $9 \cdot 2$ & $4 \cdot 5$ & $2 \cdot 5-8 \cdot 0$ & $<0 \cdot 001$ \\
\hline Not sure & $20 \cdot 5$ & $14 \cdot 5$ & $2 \cdot 1$ & $1 \cdot 1-3 \cdot 9$ & $0 \cdot 01$ \\
\hline Missing & $2 \cdot 6$ & $1 \cdot 7$ & & & \\
\hline
\end{tabular}

CI, Confidence interval.

* All odds ratios are from multiple imputation models and are adjusted for age, sex and social class; odds ratios for intermediate and proximal risk factors are adjusted for higher level variables in the conceptual framework that were included in the final model, except the presence of a baby in the household.

$\dagger P$ values are from a Wald test of regression coefficients.

$P=0 \cdot 09)$. After adjusting for age and sex, both eating salad (OR $0 \cdot 6,95 \%$ CI $0 \cdot 4-0 \cdot 8, P=0 \cdot 004$ ) and participation in water sports (OR $0 \cdot 6,95 \%$ CI $0 \cdot 4-1 \cdot 0$, $P=0.06)$ were associated with lower odds of asymptomatic norovirus infection in older children and adults. No other variables were associated with asymptomatic norovirus infection. Supplementary File 6 shows the odds ratios for the association of asymptomatic norovirus infection with the factors that were associated with norovirus-associated IID.

\section{DISCUSSION}

The major risk factors for norovirus-associated IID were related to contact with an infectious person.
Foreign travel and consumption of shellfish increased the risk of norovirus-associated IID, while consumption of raw fruit and vegetables, participation in water sports and contact with animals were associated with a decreased risk of norovirus-associated IID. We found no evidence that any exposure greatly increased the risk of asymptomatic norovirus infection, although eating salad and water sports participation were associated with a lower risk of asymptomatic infection in older children and adults.

Infectious contacts accounted for more than half of norovirus-associated IID in children aged $<5$ years and almost half in older children and adults. The importance of contact with individuals with IID for transmission of norovirus has been reported in 
Table 4. Risk of norovirus-associated IID due to the number and age of household infectious contacts in England, 1993-1996

\begin{tabular}{|c|c|c|c|c|c|c|}
\hline & \multicolumn{3}{|c|}{ Children aged $<5$ years } & \multicolumn{3}{|c|}{ Older children and adults } \\
\hline & $\mathrm{OR}^{*}$ & $95 \% \mathrm{CI}$ & $P$ value $\dagger$ & $\mathrm{OR}^{*}$ & $95 \% \mathrm{CI}$ & $P$ value $\dagger$ \\
\hline \multicolumn{7}{|l|}{ Number of household infectious contacts } \\
\hline 0 & $1 \cdot 0$ & & & $1 \cdot 0$ & & \\
\hline 1 & $1 \cdot 6$ & $0 \cdot 8-3 \cdot 1$ & $0 \cdot 15$ & $1 \cdot 4$ & $0 \cdot 8-2 \cdot 4$ & $0 \cdot 26$ \\
\hline$\geqslant 2$ & $2 \cdot 9$ & $0 \cdot 8-10 \cdot 3$ & $0 \cdot 10$ & $5 \cdot 8$ & $1 \cdot 7-19 \cdot 3$ & $0 \cdot 005$ \\
\hline \multicolumn{7}{|l|}{ Age of household infectious contact } \\
\hline No infectious contacts & $1 \cdot 0$ & & & $1 \cdot 0$ & & \\
\hline 1 or more child(ren) aged $<5$ years & $2 \cdot 6$ & $1 \cdot 0-6 \cdot 8$ & $0 \cdot 06$ & $4 \cdot 3$ & $1 \cdot 9-9 \cdot 6$ & $<0 \cdot 001$ \\
\hline Adults and children aged $\geqslant 5$ years only & $1 \cdot 6$ & $0 \cdot 8-3 \cdot 3$ & $0 \cdot 16$ & $1 \cdot 5$ & $0 \cdot 8-1 \cdot 6$ & $0 \cdot 172$ \\
\hline
\end{tabular}

OR, Odds ratio; CI, confidence interval.

* Odds ratios are from multiple imputation models and are adjusted for age, sex, social class and all other risk factors included in the final models shown in Tables 2 and 3, except a baby in the household for older children and adults and infectious contact variable.

$\dagger P$ values are from a Wald test of regression coefficients.

previous case-control studies $[6,10,12]$ and there is substantial evidence of person-to-person transmission from outbreak investigations [24-26]. While neither household size, nor crowding (the number of people per room), affected the risk of norovirus-associated IID, we found that the age of household members was a risk factor in older children and adults. Living in a household with a baby or young children accounted for a third of norovirus-associated IID in older children and adults. The highest incidence of norovirusassociated IID is in young children, so this association may indicate that they are more likely to introduce norovirus into a household than older individuals. Furthermore, when a household contact with IID symptoms was reported, the risk of norovirusassociated IID was greatest when this contact was a young child. This pattern of transmission, from young children to adults, was also observed in a large household transmission study following a pointsource norovirus outbreak [27].

However, after calculation of the PAFs, a substantial proportion of norovirus cases were not attributed to any of the recognized risk factors for norovirus transmission. It is likely that some norovirus cases failed to report contact with symptomatic individuals because of poor recall or not knowing about the symptoms experienced by individuals with whom they had contact. In addition, it is possible that environmental contamination may contribute to the transmission of norovirus leading to sporadic norovirus-associated IID, meaning that some norovirus cases in this study did not actually come into direct contact with a person with symptomatic norovirus infection, but were in contact with surfaces contaminated by infected individuals.

In young children, the risk associated with infectious contacts outside the household was much greater than that associated with infectious contacts inside the household, although we found no risk specifically associated with attendance at day care. Norovirus causes symptomatic infection, with high viral loads, in individuals of all ages [4], so there is potential for transmission to children in a wide variety of settings, not just through contact with other young children in day-care settings. We also found no protective effect of breastfeeding against norovirusassociated IID in infants. Norovirus-specific immunoglobulin A antibody has been recovered from breast milk [28], but the antigenic variation of noroviruses is complex $[29,30]$ and even strain-specific immunity is believed to last no longer than a year [31, 32].

Individuals of all ages who had recently travelled outside the UK had an increased risk of norovirusassociated IID. This risk has been demonstrated in previous studies [33], and may be attributable to changes in risk behaviours while travelling, or exposure to a different spectrum of norovirus strains. However, recent foreign travel increases the likelihood that an individual with IID due to any pathogen will present to a GP [34]; the majority of the norovirus cases in this study $(73 \%)$ were ascertained after 
presentation to a GP, rather than from the community cohort, but the prevalence of recent foreign travel was very similar in GP and community norovirus cases ( $6 \%$ and $5 \%$, respectively), so it is unlikely that this association is due to the route of case recruitment. It is possible that using cases recruited at GPs may have caused the association between social class and norovirus-associated IID in young children, because substantially more community cases were from non-manual occupational social classes (community cases $70 \% v s$. GP cases $41 \%$ ).

There was no evidence that hand washing was protective against norovirus-associated IID, despite many observational, intervention and laboratory studies showing that hand washing with soap and water is an effective method for reducing the incidence of infection with directly transmitted viruses $[35,36]$, including norovirus [35]. Hand hygiene was not a primary exposure in the IID study and was collected specifically from the person in the household responsible for food shopping and preparation (not always the study participant), only in relation to food preparation. In addition, normal hand-washing practices used by study participants may not be as rigorous as those used in experimental studies and participants may also have falsely reported good hand hygiene because this is a socially desirable response [37]. It is therefore unlikely that this variable accurately captured the general hand hygiene behaviour of study participants.

Published outbreak investigations have attributed norovirus-associated IID to contamination of food during preparation in restaurant and catering settings $[25,26,38]$, as well as to raw fruit and vegetables contaminated during wholesale production [39], consumption of oysters and other shellfish [40], and to contamination of both drinking [41, 42] and recreational water $[43,44]$. We were unable to examine drinking-water exposures, but found that water sports participation and raw fruit and vegetable consumption were actually protective against norovirusassociated IID, as was contact with animals. We found no evidence of increased risk associated with eating at restaurants or catered events. The reduced risk associated with animal contact has been reported in two previous case-control studies of communityacquired norovirus-associated IID in high-income countries $[6,10]$ and water sports were associated with a reduced risk of IID due to other pathogens in this study population [12]. There are a number of potential explanations for these exposures reducing the risk of norovirus-associated IID: (i) they are correlated with other lifestyle factors that are protective against norovirus-associated IID [12]; (ii) they do lead to norovirus transmission but are repetitive, long-term behaviours, so exposed individuals have higher levels of norovirus immunity, due to regular immune boosting; or (iii) specifically for consumption of fruit and vegetables, they have positive effects on gut immunity or the balance of intestinal bacterial flora, increasing resistance to IID [12]. Further investigation of the mechanisms underlying these associations may provide novel approaches for preventing norovirus disease. In contrast, oysters and other shellfish, which may be consumed less frequently than fruit and vegetables, and in which high-level norovirus contamination is common [45], did increase the risk of norovirus-associated IID, although they accounted for only a small proportion of norovirus disease. Finally, although foods prepared in restaurants are commonly reported as vehicles of infection in outbreaks, it is possible that breakdown in food hygiene is relatively infrequent and therefore contributes little to the overall population burden of sporadic norovirus-associated IID.

IID cases were recruited either after self-reporting an episode of IID in the community cohort or after presenting to a GP with IID; no IID cases were specifically recruited through outbreak investigations. There were no items on the risk-factor questionnaire asking about IID case involvement in a recognized IID outbreak and although this information was requested from participants by the study personnel involved in case ascertainment and recruitment, it is unfortunately incomplete for the majority of norovirus cases included in this analysis. It is therefore likely that some of the norovirus cases were infected during outbreaks. However, for a pathogen such as norovirus, which is always transmitted between infectious persons, either directly through physical contact, or indirectly via environmental or food contamination, the distinction between outbreak and sporadic cases may be artificial in relation to the transmission routes of these infections. Norovirus outbreaks arise from the same epidemiological processes as sporadic cases. This is in contrast to pathogens such as Salmonella spp. or Campylobacter spp., which are zoonotic foodborne infections with minor, secondary person-to-person spread, meaning that outbreak and sporadic cases could potentially arise from very different underlying epidemiological processes. Therefore, we believe that the inability to 
discriminate between sporadic and outbreak norovirus cases does not undermine the epidemiological rigour of the analysis.

None of the exposures that increased the risk of norovirus-associated IID were associated with asymptomatic norovirus infection. The asymptomatic norovirus infections detected in this study were prevalent, not incident, infections. Asymptomatic individuals were recruited at random from the general population and prior to determination of their norovirus infection status. It is possible that the transmission event leading to many of these asymptomatic infections occurred outside of the 10-day retrospective exposure period that was measured in the risk-factor questionnaire, and would therefore not have been captured in the responses. Norovirus has been detected by RT-PCR for at least 2 weeks after experimental inoculation in otherwise healthy adult volunteers who did not develop diarrhoea or vomiting [8]. Prolonged post-symptomatic shedding has also been demonstrated in inoculated volunteers and community cases, lasting from 1 to 8 weeks $[8,46]$. Even for those individuals who were infected during the questionnaire exposure period, if they did not collect their specimen concurrently to questionnaire completion, their norovirus infection status may not correspond to the exposures reported. Only studies with frequent and regular stool specimen collection and testing, irrespective of disease status, could ensure that proximal risk factors reported by individuals with asymptomatic norovirus infection relate to the transmission event. However, such studies are resource intensive and may be difficult to justify, in terms of the benefits to patients, or improvements in epidemiological knowledge, without first demonstrating the importance of asymptomatic infections in transmission.

This is the first study of risk factors for norovirusassociated IID to use viral load to identify norovirus cases, rather than using a positive RT-PCR result or electron microscopy detection. Previous work has shown that many IID cases that were norovirus RT-PCR positive in the IID study had the same viral loads seen in healthy controls (about $50 \%$ in this study [4]), indicating that their norovirus infection may not actually be the cause of their illness [14]. Including cases whose IID is not caused by norovirus in the analysis would have introduced misclassification with respect to the outcome. It is likely that IID cases with low norovirus loads have disease caused by a range of other gastrointestinal pathogens, so it is difficult to predict the consequences of incorrectly including these other cases in an analysis of risk factors for norovirus-associated IID. Using viral load to diagnose norovirus-associated IID substantially reduces the occurrence of this type of outcome misclassification, although it may not eliminate it completely. It is also possible that some IID cases with disease caused by norovirus were incorrectly excluded from this analysis because they had viral loads below the cut-off, due to specimen collection after symptom resolution (when norovirus viral loads quickly decrease [8]) or because the sensitivity of the cut-off is not $100 \%$. However, this type of outcome misclassification is unlikely to have affected the results of this analysis unless the incorrectly excluded norovirus cases differed systematically from those norovirus cases that were included in the analysis.

While we were able to use a published viral load cut-off for classifying norovirus aetiology in genogroup II-infected IID cases, there is no published cut-off for norovirus genogroup I. We therefore limited the inclusion criteria for genogroup I norovirus-infected IID cases to those that were positive by electron microscopy and subsequently confirmed by real-time RT-PCR. This does mean that genogroup I norovirus cases were underrepresented in the analysis, but comparison of risk factors between the genogroups was not the aim of the analysis, and even if a genogroup I cut-off had been available, it is unlikely that there would have been sufficient numbers of genogroup I-infected norovirus cases and controls for such a comparison. There have been no studies comparing risk factors between norovirus genogroups or genotypes, so it is unclear what effect, if any, grouping them together in the analysis might have had on the results. However, evidence from outbreaks indicates that genogroup I and genogroup II noroviruses are both directly transmissible, via person-to-person contact, and both genogroups have also been detected in food- and water-borne outbreaks $[25,26,41]$. The only currently recognized, notable difference between norovirus genotypes is the human blood group antigen (HBGA) binding specificity required for host cell infection [47]. HBGA phenotype was not determined for the individuals included in this study, but it is possible that some of the norovirus negative controls used as the comparison group in the analysis were not actually susceptible to infection with some norovirus genotypes, although it is unlikely that they would be resistant to all norovirus genotypes. 
The results of our analysis have corroborated evidence from previous studies about the importance of person-to-person transmission in the acquisition of norovirus-associated IID. Experimental virus transfer and epidemiological studies indicate that good hand hygiene and cleaning of environmental surfaces with appropriate products can decrease norovirus transmission [35, 48, 49]. Further reinforcement of existing public health messages regarding hand and domestic environmental hygiene could facilitate a reduction in norovirus transmission, although, given the low infectious dose [50] and the current lack of any sanitizers that completely inactivate norovirus, reductions in sporadic disease incidence may be limited.

\section{NOTE}

Supplementary material accompanies this paper on the Journal's website (http://journals.cambridge. org/hyg).

\section{ACKNOWLEDGEMENTS}

The authors acknowledge the help of the following people: Jim Gray, Miren Iturriza-Gomara, Corrine Amar, Fenella Halstead, Dalia Choudhury and Mihaela Cirdei for completing the laboratory work; Julian Gardiner, Patrick Royston and Ian White for advice on the multiple imputation; Ian White for providing Stata code.

\section{DECLARATION OF INTEREST}

None.

\section{REFERENCES}

1. de Wit MA, et al. Sensor, a population-based cohort study on gastroenteritis in the Netherlands: incidence and etiology. American Journal of Epidemiology 2001; 154: 666-674.

2. Hellard ME, et al. A randomized, blinded, controlled trial investigating the gastrointestinal health effects of drinking water quality. Environmental Health Perspectives 2001; 109: 773-778.

3. Marshall JA, et al. Incidence and characteristics of endemic Norwalk-like virus-associated gastroenteritis. Journal of Medical Virology 2003; 69: 568-578.

4. Phillips G, et al. Community incidence of norovirusassociated infectious intestinal disease in England: improved estimates using viral load for norovirus diagnosis. American Journal of Epidemiology 2010; 171: 1014-1022.
5. Phillips G, et al. Prevalence and characteristics of asymptomatic norovirus infection in the community in England. Epidemiology and Infection 2010; 138: 1454-1458.

6. Karsten C, et al. Incidence and risk factors for community-acquired acute gastroenteritis in northwest Germany in 2004. European Journal of Clinical Microbiology \& Infectious Diseases 2009.

7. Marshall JA, et al. Failure to detect norovirus in a large group of asymptomatic individuals. Public Health 2004; 118: $230-233$.

8. Atmar RL, et al. Norwalk virus shedding after experimental human infection. Emerging Infectious Diseases 2008; 14: 1553-1557.

9. Lindesmith $\mathbf{L}$, et al. Human susceptibility and resistance to Norwalk virus infection. Nature Medicine 2003; 9: 548-553.

10. de Wit MA, Koopmans MP, Van Duynhoven YT. Risk factors for norovirus, Sapporo-like virus, and group A rotavirus gastroenteritis. Emerging Infectious Diseases 2003; 9: 1563-1570.

11. Fretz R, et al. Risk factors for infections with Norovirus gastrointestinal illness in Switzerland. European Journal of Clinical Microbiology \& Infectious Diseases 2005; 24: 256-261.

12. Food Standards Agency. A report of the study of infectious intestinal disease in England. London: HMSO, 2000 .

13. Amar CF, et al. Detection by PCR of eight groups of enteric pathogens in 4,627 faecal samples: reexamination of the English case-control Infectious Intestinal Disease Study (1993-1996). European Journal of Clinical Microbiology \& Infectious Diseases 2007; 26: 311-323.

14. Phillips G, et al. Diagnosing norovirus-associated infectious intestinal disease using viral load. $B M C$ Infectious Diseases 2009; 9: 63.

15. Tompkins DS, et al. A study of infectious intestinal disease in England: microbiological findings in cases and controls. Communicable Disease and Public Health 1999; 2 : 108-113.

16. Sethi D, et al. A study of infectious intestinal disease in England: plan and methods of data collection. Communicable Disease and Public Health 1999; 2: 101-107.

17. Haruki K, et al. Pattern of shedding of small, roundstructured virus particles in stools of patients of outbreaks of food-poisoning from raw oysters. Microbiology and Immunology 1991; 35: 83-86.

18. Thornhill TS, et al. Pattern of shedding of the Norwalk particle in stools during experimentally induced gastroenteritis in volunteers as determined by immune electron microscopy. Journal of Infectious Diseases 1975; 132: 28-34.

19. Victora CG, et al. The role of conceptual frameworks in epidemiological analysis: a hierarchical approach. International Journal of Epidemiology 1997; 26: 224 227.

20. Bland JM, Altman DG. Matching. British Medical Journal 1994; 309: 1128. 
21. Royston P. Multiple imputation of missing values: update of ice. Stata Journal 2005; 5: 527-536.

22. StataCorp. Stata Statistical Software, release 10 [computer program]. College Station, TX: Stata Corporation LP; 2007.

23. Brady T. Adjusted population attributable fractions from logistic regression. Stata Technical Bulletin 2009; 42: $8-12$.

24. Dedman D, et al. Surveillance of small round structured virus (SRSV) infection in England and Wales, 1990-5. Epidemiology and Infection 1998; 121: 139-149.

25. Fretz R, et al. Outbreaks of gastroenteritis due to infections with Norovirus in Switzerland, 2001-2003. Epidemiology and Infection 2005; 133: 429-437.

26. Kroneman A, et al. Analysis of integrated virological and epidemiological reports of norovirus outbreaks collected within the foodborne viruses in Europe Network from 1 July 2001 to 30 June 2006. Journal of Clinical Microbiology 2008; 46: 2959-2965.

27. Gotz H, et al. Clinical spectrum and transmission characteristics of infection with Norwalk-like virus: findings from a large community outbreak in Sweden. Clinical Infectious Diseases 2001; 33: 622-628.

28. Makita K, et al. First detection of $\operatorname{IgA}$ against norovirus in breast milk. Clinical Laboratory 2007; 53: 125-128.

29. Hale AD, et al. Homotypic and heterotypic IgG and IgM antibody responses in adults infected with small round structured viruses. Journal of Medical Virology 1998; 54: 305-312.

30. Lindesmith LC, et al. Heterotypic humoral and cellular immune responses following Norwalk virus infection. Journal of Virology 2009.

31. Cukor G, Nowak NA, Blacklow NR. Immunoglobulin $\mathrm{M}$ responses to the Norwalk virus of gastroenteritis. Infection and Immunity 1982; 37: 463-468.

32. Parrino TA, et al. Clinical immunity in acute gastroenteritis caused by Norwalk agent. New England Journal of Medicine 1977; 297: 86-89.

33. Chapin AR, et al. Prevalence of norovirus among visitors from the United States to Mexico and Guatemala who experience traveler's diarrhea. Journal of Clinical Microbiology 2005; 43: 1112-1117.

34. Tam CC, Rodrigues LC, O'Brien SJ. The study of infectious intestinal disease in England: what risk factors for presentation to general practice tell us about potential for selection bias in case-control studies of reported cases of diarrhoea. International Journal of Epidemiology 2003; 32: 99-105.

35. Barker J, Vipond IB, Bloomfield SF. Effects of cleaning and disinfection in reducing the spread of Norovirus contamination via environmental surfaces. Journal of Hospital Infection 2004; 58: 42-49.

36. Ejemot RI, et al. Hand washing for preventing diarrhoea. Cochrane Database of Systematic Reviews 2008; Issue No. 1. Art No. CD004265.
37. Surgeoner BV, Chapman BJ, Powell DA. University students' hand hygiene practice during a gastrointestinal outbreak in residence: what they say they do and what they actually do. Journal of Environmental Health 2009; 72: 24-28.

38. Baert L, et al. Reported foodborne outbreaks due to noroviruses in Belgium: the link between food and patient investigations in an international context. Epidemiology \& Infection 2009; 137: 316-325.

39. Korsager B, et al. Two outbreaks of norovirus infections associated with the consumption of imported frozen raspberries, Denmark, May-June 2005. Eurosurveillance 2005; 10: E050623.

40. Kingsley DH, Meade GK, Richards GP. Detection of both hepatitis A virus and Norwalk-like virus in imported clams associated with food-borne illness. Applied and Environmental Microbiology 2002; 68: 3914-3918.

41. Maunula L, Miettinen IT, von Bonsdorff CH. Norovirus outbreaks from drinking water. Emerging Infectious Diseases 2005; 11 : 1716-1721.

42. Yoder J, et al. Surveillance for waterborne disease and outbreaks associated with drinking water and water not intended for drinking - United States, 2005-2006. Morbidity and Mortality Weekly Reports. Surveillance Summaries 2008; 57: 39-62.

43. Maunula L, et al. Wading pool water contaminated with both noroviruses and astroviruses as the source of a gastroenteritis outbreak. Epidemiology and Infection 2004; 132: 737-743.

44. Podewils LJ, et al. Outbreak of norovirus illness associated with a swimming pool. Epidemiology and Infection 2006; 135: 827-833.

45. Cheng PK, et al. Norovirus contamination found in oysters worldwide. Journal of Medical Virology 2005; 76: 593-597.

46. Rockx B, et al. Natural history of human calicivirus infection: a prospective cohort study. Clinical Infectious Diseases 2002; 35: 246-253.

47. Huang $\mathbf{P}$, et al. Norovirus and histo-blood group antigens: demonstration of a wide spectrum of strain specificities and classification of two major binding groups among multiple binding patterns. Journal of Virology 2005; 79: 6714-6722.

48. Chimonas MA, et al. Passenger behaviors associated with norovirus infection on board a cruise shipAlaska, May to June 2004. Journal of Travel Medicine 2008; 15: 177-183.

49. Magulski T, et al. Inactivation of murine norovirus by chemical biocides on stainless steel. BMC Infectious Diseases 2009; 9: 107.

50. Teunis PF, et al. Norwalk virus: how infectious is it? Journal of Medical Virology 2008; 80 : 1468-1476.

51. Thomas R, Elias P. Development of the standard occupational classification. Population Trends 1989; 55: 16-21. 